\title{
Cerebral palsy in SA: Perinatal factors and later neurological handicap in infants born at or near term
}

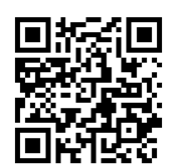

It has been recognised in high-income countries for many years that most cases of cerebral palsy are not due to complications of labour and delivery. In a report published in 2004, Jacobsson and Hagberg ${ }^{[1]}$ concluded that $70-80 \%$ of cases of cerebral palsy were due to prenatal factors, whereas birth asphyxia accounted for $<10 \%$ of cases. This was reiterated in a recent review by Nelson and Blair ${ }^{[2]}$ who quoted other studies that have produced similar figures.However, these studies have been confined to high-income countries and are unlikely to be applicable to low- and middle-income countries (LMICs).

The target year for the Millennium Development Goals (MDGs), 2015 , has been reached. One of these goals was to reduce mortality of infants and children under the age of 5 years by two-thirds, with the figure for 1990 as the baseline. While this goal was not reached globally, a reduction of just over 50\% was achieved - an impressive amount. However, neonatal mortality rates have declined more slowly than postneonatal mortality, with the result that, globally, $44 \%$ of all under-5 deaths occur in the neonatal period, of which onequarter are owing to intrapartum-related factors, i.e. intrapartum hypoxia or what is commonly referred to as birth asphyxia. ${ }^{[3]}$ The vast majority of these deaths occur in LMICs. South African (SA) data are similar; hypoxia-related deaths make up $28 \%$ of all early neonatal deaths. However, when only infants with birth weight $>1000 \mathrm{~g}$ are analysed, hypoxia-related deaths make up $41 \%$ of all early neonatal deaths, the single largest category of cause of death. ${ }^{[4]}$

While accurate figures for intrapartum-related causes of cerebral palsy in SA are not known, with this large number of deaths due to intrapartum hypoxia, the number of infants surviving such an insult with neurological damage is likely to be substantially higher than that given for high-income countries. This conclusion was supported by a retrospective study by Mahlaba (J Rodda and N Mahlaba, personal communication), who reviewed children attending the cerebral palsy clinic at Chris Hani Baragwanath Academic Hospital. Of the 144 cases reviewed, 88 had neuroimaging reports available and $42 \%$ of these showed findings compatible with a hypoxic ischaemic injury. Imaging results were not available for the other cases, but the above example still illustrates that intrapartum hypoxia is a significantly more common cause of cerebral palsy compared with high-income countries.

Ascribing subsequent cerebral palsy to intrapartum events has been the subject of many publications in the literature. A consensus statement on this was published by MacLennan ${ }^{[5]}$ in 1999, which was supported by national obstetric, paediatric and midwifery organisations from a number of countries.According to this consensus statement, three essential requirements needed to be fulfilled before it could be concluded that later handicap could be attributed to intrapartum events, namely: (i) severe metabolic acidosis demonstrated on cord blood or blood taken from the neonate within 1 hour of birth; (ii) moderate or severe neonatal encephalopathy; and (iii) cerebral palsy of the spastic quadriplegic or dyskinetic type. These criteria could only be applied when the gestation was $\leq 34$ weeks. As knowledge was gained from brain magnetic resonance imaging (MRI) of infants who had well-documented intrapartum hypoxic events, additional imaging criteria were added. However, it became clear that these criteria were too rigid and that lesser degrees of acidosis could still be associated with neurological damage occurring intrapartum, and that there was a spectrum of neurological handicap, including other forms of cerebral palsy, that could - albeit much less commonly - result from intrapartum hypoxia. However, moderate or severe neonatal encephalopathy remains the sine qua non for linking intrapartum events to later handicap. In view of this, the American College of Obstetricians and Gynecologists (ACOG) recently published an updated report, endorsed by the American Academy of Pediatrics, on the relationship between neonatal encephalopathy and neurological outcome. The report stressed that all relevant factors should be taken into account to evaluate the likelihood of such a relationship, rather than the more rigid requirement for essential criteria as outlined by the MacLennan consensus. The ACOG report acknowledged that there were still significant knowledge gaps, that neurological injury may occur at multiple points during gestation and that these insults occurring at different times may be additive. ${ }^{[6]}$

In recent years, SA has seen a major increase in litigation involving those responsible for the care of women during labour and delivery, with payouts of many millions of rand (ZAR) for neurological damage in the child. This has occurred in both the public and the private sector. From the private sector perspective, the result has been that, similar to what has happened in a number of other countries, obstetricians now face prohibitive rates for malpractice insurance. With regard to the public sector, grave concern has been expressed that the extent of this litigation is threatening to have a major financial effect on the public healthcare system. More recently, the actions of those responsible for resuscitation of the neonate and subsequent ongoing management have also become the focus of litigation.

In addition to healthcare worker issues, systems failure - especially in the public sector - also plays an important role. There may be long delays in transporting a mother from a primary care facility to one able to expedite delivery. In other cases, significant delays from the time the decision is taken to do a caesarean section and the actual time of delivery frequently occur due to heavy patient load.

It is therefore clear that SA has a major problem not only with a high neonatal mortality due to intrapartum and immediate postnatal events, but also with respect to neurological handicap in survivors. Additional training of healthcare workers at all levels is essential for the care of mothers during pregnancy, labour and delivery as well as for neonatal resuscitation and early neonatal care. Measures need to be put in place to ensure that the healthcare system is able to provide for efficient and seamless care of mothers and babies across all levels of care and that adequate resources are in place to cope with heavy patient load.

\section{References}

1. Jacobsson B, Hagberg G. Antenatal risk factors for cerebral palsy. Best Pract Res Clin Obstet Gynecol 2004;18(3):425-436. [http://dx.doi.org/10.1016/j. bpobgyn.2004.02.011]

2. Nelson KB, Blair E. Prenatal factors in singletons with cerebral palsy born at or near term. N Engl J Med 2015;373(10):946-953. [http://dx.doi.org/10.1056/nejmra1505261]

3. UNICEF, World Health Organization. Every Newborn: An Action Plan to End Preventable Deaths. 2014. http://www.everynewborn.org/Documents/Fullaction-plan-EN.pdf (accessed 2 November 2015).

4. Pattinson R, Rhoda N. Saving babies 2012 - 2113: Ninth report on perinatal care in South Africa. http://www.ppip.co.za/wp-content/uploads/SavingBabies-2012-2013.pdf (accessed 2 November 2015).

5. MacLennan A. A template for defining a causal relationship between acute intrapartum events and cerebral palsy: International consensus statement. BM] 1999;319(7216):1054-1059. [http://dx.doi.org/10.1136/bmj.319.7216.1054]

6. Neonatal encephalopathy and neurological outcome, second edition. Pediatrics 2014;133(5);e1482-1488. [http://dx.doi.org/10.1542/peds.2014-0724]

\section{Prof. P Cooper}

Academic Head, Department of Paediatrics and Child Health, Faculty of Health Sciences, University of the Witwatersrand; and Chief Paediatrician, Charlotte Maxeke Johannesburg Academic Hospital

S Afr J Child Health 2015;9(4):107. DOI:10.7196/SAJCH.2015.v9i4.1064 